\title{
Long non-coding nuclear paraspeckle assembly transcript 1 acts as prognosis biomarker and increases cell growth and invasion in cervical cancer by sequestering microRNA-101
}

\author{
LI WANG $^{1}$ and HONGYING ZHU ${ }^{2}$ \\ ${ }^{1}$ Department of Obstetrics and Gynecology, Xidian Group Hospital, Xi'an, Shaanxi 710077; \\ ${ }^{2}$ Department of Obstetrics and Gynecology, Xianyang Caihong Hospital, Xianyang, Shaanxi 712021, P.R. China
}

Received August 24, 2017; Accepted November 9, 2017

DOI: $10.3892 / \mathrm{mmr} .2017 .8186$

\begin{abstract}
Emerging studies have focused on the essential role of long non-coding RNAs (lncRNAs) in cervical carcinogenesis. A recent study has indicated that nuclear paraspeckle assembly transcript 1 (NEAT1) is highly expressed in the human cervical tissue. However, whether NEAT1 is involved in cervical cancer remains to be elucidated. Reverse transcription-quantitative polymerase chain reaction results demonstrated that the expression of NEAT1 was higher in cervical cancer cells/tissues compared with that in normal human keratinocytes/tissues. Patients with higher NEAT1 level had poorer clinical characteristics and a shorter survival time compared with those that exhibited lower NEAT1 expression levels. In vitro, flow cytometery analysis revealed that transfection with NEAT1 small interfering RNA retarded cervical cancer cell (Caski and HeLa) growth by decreasing the percentage of $\mathrm{S}$ phase in the cell cycle and inducing cell apoptosis. In addition, the colony formation assay, wound healing assay and matrigel invasion assay results indicated that downregulation of NEAT1 inhibited colony formation, cell migration and invasion. Further investigation using the luciferase reporter assay revealed that the expression of mircoRNA-101 (miR-101) target gene Fos was positively associated with NEAT1 expression due to NEAT1-competitive molecular sequestering of miR-101 via base pairing. Furthermore, reduction of miR-101 expression by inhibitor transfection reversed the effect of NEAT1 siRNA on cervical cancer cells. To conclude, the present data indicated that NEAT1 promoted cervical cancer progression by targeting miR-101.
\end{abstract}

Correspondence to: Dr Hongying Zhu, Department of Obstetrics and Gynecology, Xianyang Caihong Hospital, 3 West Weiyang Road, Xianyang, Shaanxi 712021, P.R. China

E-mail: zhuhongying97@163.com

Key words: cervical cancer, long non-coding RNAs, that nuclear paraspeckle assembly transcript 1 , microRNA-101

\section{Introduction}

Human cervical cancer is one of the most common and lethal malignancies in women worldwide $(1,2)$. Despite the fact that cervical cancer incidence has remarkably reduced with the application of Papanicolaou smear screening, cervical cancer remains imperil women's health, with an estimated 275,000 deaths annually $(3,4)$. Regarding the etiology of cervical cancer, there's evidence that cervical cancer is associated with persistent oncogenic human papillomavirus (HPV) infections inducing atypical hyperplasia and abnormal maturation leading to cervical intraepithelial neoplasia (CIN) (5). In reality, the development of cervical cancer is a lengthy course from CIN1 to CIN3. Although the cure rate for cervical cancer is up to $80-90 \%$ in the early stage, the prognosis of patients with advanced stage or recurrence is still poor (6). Therefore, it is necessary to uncover the molecular mechanism and to identify effective prognostic markers in cervical cancer.

Long non-coding RNAs (lncRNAs), a cluster of RNAs longer than 200 nucleotides, are important member of the ncRNA family, yielding little or no protein (7). It is proved that lncRNA play important roles in physiological and pathological processes, including genome imprinting, gene transcription, cellular differentiation and immune response $(8,9)$. Emerging evidence has been shown that lncRNA modulate gene activity in response to external oncogenic stimuli and DNA damage, indicating the potential involvement of lncRNAs in the pathogenesis of human diseases, especially in cancer (10). Recently, accumulating evidences show that aberrantly expressed lncRNAs could be associated with cervical cancer progression $(11,12)$. Understanding of molecular mechanisms of lncRNAs in cervical cancer process may be greatly valuable for cervical cancer therapy. Recent studies indicate that aberrant expression of lncRNA nuclear paraspeckle assembly transcript 1 (NEAT1) has been documented in different types of solid tumors (including lung cancer (13), oesophageal cancer (14), colorectal cancer (15) and hepatocellular carcinoma (16). However, whether NEAT1 is involved in cervical cancer remains to be elucidated.

In the current study, we aimed to explore the expression and clinical significance of NEAT1 in cervical cancer. 
Firstly, we demonstrated that overexpression of NEAT1 was found in cervical cancer, and its level was associated with prognosis. Next, we downregulated the NEAT1 in HeLa/Caski cells to assess the effect of NEAT1 on cervical cancer cell growth, cell cycle, apoptosis, migration and invasion. Then, the molecular mechanisms underlying NEAT1 in cervical cancer were investigated. Our findings suggest that NEAT1 serves as an important role in cervical cancer development.

\section{Materials and methods}

Patients and clinical samples. Human cervical cancer specimens and adjacent normal tissues were obtained from 68 patients admitted to Department of Obstetrics and Gynecology, Xidian Group Hospital (Xi'an, China) between August 2013 and June 2016. All included patients did not accept any local or systemic treatment. The samples were determined by pathologic examination and immediately placed in liquid nitrogen followed by storage at $-80^{\circ} \mathrm{C}$ after surgical resection. This study was approved by the Ethics Review Board of Department of Obstetrics and Gynecology, Xidian Group Hospital. Written informed consent was gathered from all participants.

RNA extraction and cDNA synthesis. Total RNA from clinical samples and cells were extracted using TRIzol reagent (Takara Bio, Inc., Otsu, Japan) according to the manufacturer's instructions. Concentration and purification $(1.8<$ OD $260 / 280<2.0)$ of RNA were detected using a NanoDrop ND-1000 spectrophotometer (Thermo Fisher Scientific, Inc., Waltham, MA, USA). Then, the agarose gel electrophoresis was used to determine RNA integrity. cDNA was generated from $1 \mu \mathrm{g}$ RNA template using Reverse Transcription kit (Invitrogen; Thermo Fisher Scientific, Inc.).

Quantitative polymerase chain reactions ( $q P C R$ ) expression assay. qPCR was performed in a volume of $30 \mu \mathrm{l}$ using SYBR-Green PCR kit protocol in the ABI StepOnePlus System (Applied Biosystems; Thermo Fisher Scientific, Inc.). PCR cycling process was set as follows: initial denaturation at $98^{\circ} \mathrm{C}$ for $5 \mathrm{~min} ; 35$ cycles of denaturation at $95^{\circ} \mathrm{C}$ for $15 \mathrm{sec}$, annealing at $55^{\circ} \mathrm{C}$ for $1 \mathrm{~min}$, and elongation at $72^{\circ} \mathrm{C}$ for $1 \mathrm{~min}$; and a final elongation at $72^{\circ} \mathrm{C}$ for $10 \mathrm{~min}$. U6 snRNA was used for normalization and $2^{-\Delta \Delta \mathrm{Cq}}$ method was used to determine the relative expression of NEAT1.

Cell culture and transfection. Cervical cancer cell lines SiHa, Caski, HeLa were purchased from Institute of Biochemistry and Cell Biology at the Chinese Academy of Sciences (Shanghai, China). SiHa cells were cultured in Dulbecco's modified Eagle's medium (DMEM; Gibco, Shanghai, China) containing $10 \%$ fetal bovine serum (FBS; Gibco). Caski and HeLa cells were grown in RPMI-1640 medium (Gibco) supplemented with $10 \%$ FBS. All cells were incubated under $5 \% \mathrm{CO}_{2}$, saturated humidity at $37^{\circ} \mathrm{C}$ for 3 days. MicroRNA (miRNA/miR) mimic/inhibitor and si-lncRNA were designed and synthesized by GenePharma (Shanghai, China). For cell transfection, cells were seeded into 24-well plates and were transfected with miRNA or siRNA (20 nM) with
Lipofectamine ${ }^{\circledR} 2000$ (Invitrogen; Thermo Fisher Scientific, Inc.) was used for transfection according to the manufacturer's instructions when cell density reached $60-70 \%$ confluence. Cells were harvested and subjected to subsequent analyses after $48 \mathrm{~h}$ transfection.

Plasmid construction and luciferase reporter assay. Potential wild type and mutant binding sequence of NEAT1 was synthesized by PCR-amplification and cloned into the pmirGLO vector (Promega Corporation, Madison, WI, USA). Cells were placed into 24-well plates and were cotransfected with wild type/mutant pmirGLO-NEAT1 and miRNA using Lipofectamine ${ }^{\circledR} 2000$ (Invitrogen; Thermo Fisher Scientific, Inc.). Cells were collected and lysed with passive lysis buffer for luciferase detection by Dual-Luciferase Reporter Assay System (Promega Corporation) $48 \mathrm{~h}$ after transfection.

Cell proliferation assay. Cell viability was measured using Cell Counting Kit-8 (CCK-8; Dojindo, Tokyo, Japan) following the manufacturer's instructions. Briefly, $1 \times 10^{3}$ cells/well cells were seeded onto 96-well plates, and the cell viability was measured every $24 \mathrm{~h}$. The absorbance was detected at $450 \mathrm{~nm}$ by using an ELx 800 Microplate Reader (Bio-Tek Instruments Inc., Winooski, VT, USA).

Flow cytometry analysis. Cell cycle and apoptosis were measured by flow cytometry FACSDiva (BD Biosciences, San Jose, CA, USA). For cell cycle, cells were gathered and fixed with $70 \%$ ethanol at $-20^{\circ} \mathrm{C}$ overnight. Then, cells were stained propidium iodide by the CycleTEST PLUS DNA Reagent Kit (BD Biosciences) according to the manufacturer's introduction. Cell apoptosis was detected using double staining with propidium iodide and Annexin V-FITC from fluorescein Apoptosis kit (BD Biosciences) according to the manufacturer's introduction. Data analysis was performed using FlowJo software (Tree Star, Inc., Ashland, OR, USA).

Colony-forming assay and matrigel invasion assay. The transfected cells were seeded into 6-well plates at density of $1 \times 10^{3}$ cells/well. After cultured for 10 days, cells were fixed with $10 \%$ paraformaldehyde and were stained with $0.1 \%$ crystal violet solution (Beijing Solarbio Science \& Technology Co., Ltd., Beijing, China) followed by counting the number of colonies. For invasion assay, matrigel invasion chamber (pore size: $8 \mu \mathrm{m}, 24$-well; BD Biosciences, Bedford, MA, USA) was used. Briefly, a total of $1 \times 10^{5}$ transfected cells were seeded in the upper wells of chambers with DMEM containing $0.1 \%$ FBS. Normal medium (10\% FBS) was added to the lower chamber. $24 \mathrm{~h}$ later, the invasive cells were stained with $2 \%$ crystal violet (Sigma-Aldrich; Merck KGaA, Darmstadt, Germany) and counted in 5 high-power fields under the microscopic fields.

Wound healing assay. Transfected cervical cancer cells were grown in 12-well plates when cell density reached $90 \%$ confluence. A sterile $200 \mu \mathrm{l}$ pipette tip was used to scratch the cell monolayer. The Cells were washed with phosphate-buffered saline to remove cell debris followed by cultured in medium with $2 \%$ FBS. The wound distance was measured at $0,24 \mathrm{~h}$ using a microscope. 
Statistical analysis. Statistical analyses were conducted using SPSS version 17.0 software (SPSS, Inc., Chicago, IL, USA). All the data were expressed as mean \pm standard deviation and derived from at least three independent experiments. Pearson's $\chi^{2}$ test was used to assess the associations between NEAT1 expression and the clinicopathological characteristics. The correlation between the expression of NEAT1 and miR-101 level was determined by Spearman's correlation analysis. Student's t-test and one-way ANOVA were performed to compare the differences between two groups. Tukey's test was used when it was necessary following a one-way ANOVA. $\mathrm{P}<0.05$ was considered to indicate a statistically significant difference.

\section{Results}

Association between NEAT1 expression and clinicopathologic factors in cervical cancer. qPCR was performed to detect the expression of NEAT1 in human cervical cancer cell lines (SiHa, Caski and HeLa) and human keratinocyte cell line HaCaT. The expression of NEAT1 was observed to be significantly upregulated in cervical cancer cells compared with $\mathrm{HaCaT}$ cells (Fig. 1A). Similarly, NEAT1 expression in cervical cancer tissues was higher than that in noncancerous tissues (Fig. 1B). To evaluate the correlation between NEAT1 and clinicopathologic characteristics in cervical cancer, patients were divided into low $(n=34)$ and high $(n=34)$ groups according to NEAT1 expression. We found that NEAT1 level was positively associated with unfavorable factor, including size of carcinoma $(\mathrm{P}=0.0014)$, FIGO stage $(\mathrm{P}=0.0036)$, depth of cervical invasion $(\mathrm{P}=0.0034)$ and lymphatic metastasis $(\mathrm{P}=0.0023)$ (Table I). In addition, cervical cancer patients with high NEAT1 expression had significantly shorter overall $(\mathrm{P}=0.0245)$ and disease-free $(\mathrm{P}=0.0353)$ survival time than those with low NEAT1 expression (Fig. 1C and D).

Downregulation of NEAT1 decreases cervical cancer cells proliferation and induces cell apoptosis. Given that the aberrant overexpression of NEAT1 in cervical cancer, we investigate the function of NEAT1 on cell proliferation, apoptosis, colony-forming ability, migration and invasion by transfection with NEAT1 siRNA. The suppression efficiency in cells transfected with NEAT1 siRNAs is shown in Fig. 2A. CCK-8 assay indicated that NEAT1 knockdown significantly repressed the cell growth and colony-forming capacity (Fig. 2B and C). Moreover, we assessed the cell cycle and cell apoptosis after transfection. Flow cytometry assay showed that downregulation of NEAT1 in cervical cancer cells obviously decreased the percentage of S phase and induced apoptosis compared with the control group (Fig. 2D and E).

NEAT1 knockdown inhibits cervical cancer migration and invasion. To explore the effect of NEAT1 on migration and invasion, we performed wound healing and Matrigel invasion assays, respectively. Wound healing assay indicated that downregulation of NEAT1 obviously suppressed the width of wound closure (Fig. 2F). Similarly, following the knockdown of NEAT1 expression, the number of invasive cells was reduced compared with the control (Fig. 2G).
Table I. Correlations between NEAT1 expression in cervical cancer and clinical characteristics.

\begin{tabular}{|c|c|c|c|c|}
\hline \multirow[b]{2}{*}{ Group } & \multirow[b]{2}{*}{ No. } & \multicolumn{2}{|c|}{$\begin{array}{c}\text { Relative } \\
\text { NEAT1 } \\
\text { expression }\end{array}$} & \multirow[b]{2}{*}{ P-value } \\
\hline & & Low & High & \\
\hline \multicolumn{5}{|l|}{ Age } \\
\hline$>50$ & 36 & 20 & 16 & 0.3311 \\
\hline$\leqq 50$ & 32 & 14 & 18 & \\
\hline \multicolumn{5}{|c|}{ Size of carcinoma $(\mathrm{cm})$} \\
\hline$>4$ & 29 & 8 & 21 & 0.0014 \\
\hline$\leqq 4$ & 39 & 26 & 13 & \\
\hline \multicolumn{5}{|l|}{ Differentiation } \\
\hline Well and moderate & 37 & 22 & 15 & 0.0883 \\
\hline Poor & 31 & 12 & 19 & \\
\hline \multicolumn{5}{|l|}{ Histology } \\
\hline Squamous & 50 & 27 & 23 & 0.2716 \\
\hline Adenocarcinoma & 18 & 7 & 11 & \\
\hline \multicolumn{5}{|l|}{ FIGO stage } \\
\hline Ib-IIa & 36 & 24 & 12 & 0.0036 \\
\hline IIb-IIIa & 32 & 10 & 22 & \\
\hline \multicolumn{5}{|c|}{ Depth of cervical invasion } \\
\hline$>2 / 3$ & 30 & 9 & 21 & 0.0034 \\
\hline$\leqq 2 / 3$ & 38 & 25 & 13 & \\
\hline \multicolumn{5}{|l|}{ Lymphatic metastasis } \\
\hline No & 44 & 28 & 16 & 0.0023 \\
\hline Yes & 24 & 6 & 18 & \\
\hline
\end{tabular}

NEAT1, nuclear paraspeckle assembly transcript 1; FIGO, International Federation of Gynecology and Obstetrics.

NEAT1 functions as a sponge for miR-101 in cervical cancer cells. It was elucidated that lncRNAs serve as 'molecular sink' to regulate the biological functions of miRNAs (17). In order to identify the potential miRNA interacting with NEAT1, bioinformatics method (starBase v2.0 algorithm, http://starbase.sysu.edu.cn/index.php) was used to predict the miRNA. The results showed that miR-101 could directly bind with NEAT1. Then, qPCR was conducted after bioinformatics analyses. The results demonstrated that there is a significant negative correlation between NEAT1 and miR-101 in clinical specimens (Fig. 3A). Next, we measured the levels of miR-101 expression in Caski and HeLa cells after transfection with NEAT1 siRNA. Compared with controls, miR-101 expression was remarkably increased in cells transfected with NEAT1 siRNA (Fig. 3B). As expected, downregulation of NEAT1 inhibited Fos level that is a target gene of miR-101 (Fig. 3C). For further confirmation, we cloned the wild type/mutant fragments including the paired bases into a pmiR-GLO vector (Fig. 3D). Luciferase reporter assay indicated that overexpression of miR-101 significantly reduced the luciferase activity of wild type pmirGLO-NEAT1, but not of mutant pmirGLO-NEAT1 (Fig. 3E). 

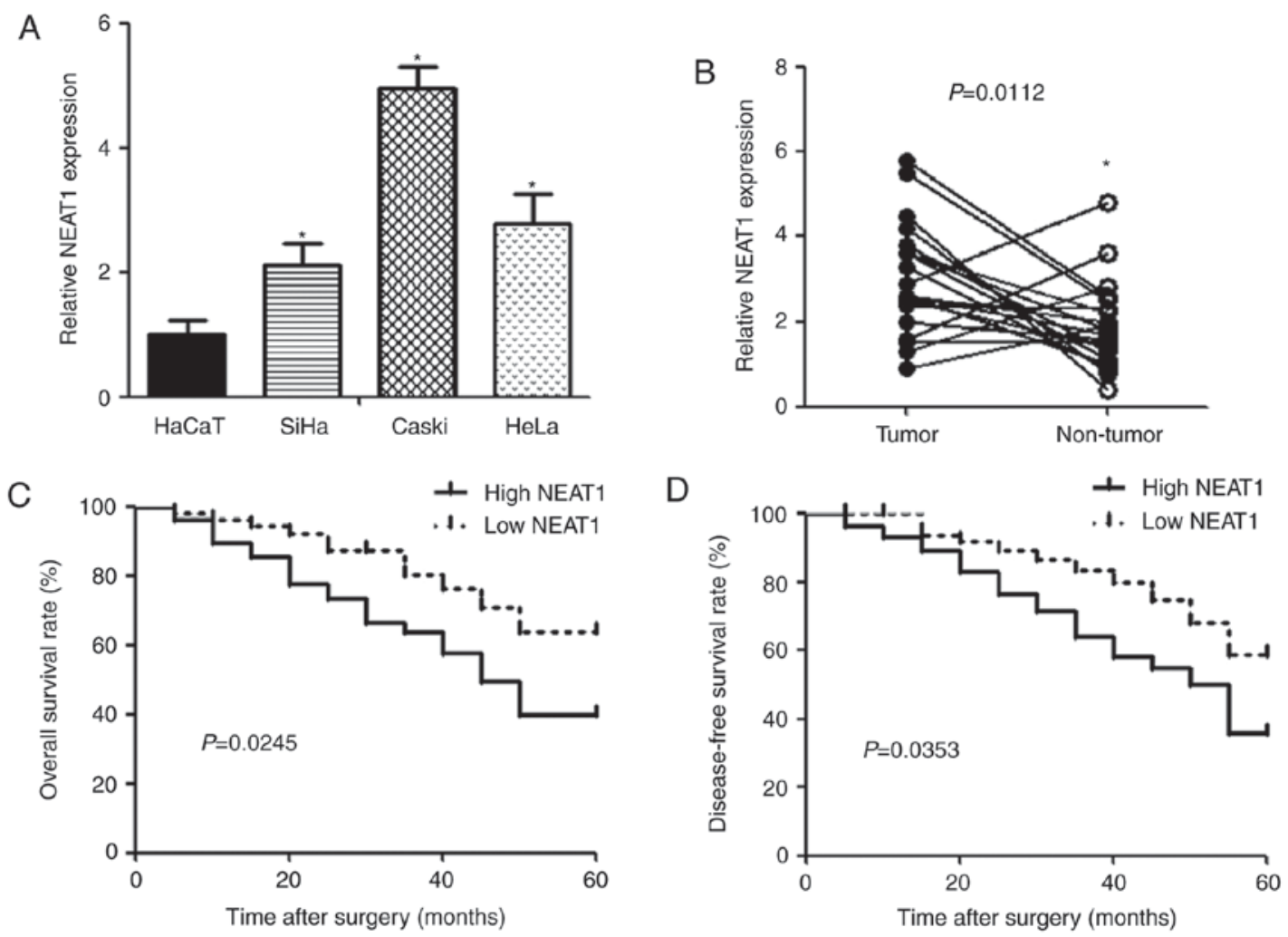

Figure 1. Relative NEAT1 expression and its clinical significance. (A) Relative NEAT1 expression was determined using qPCR in HaCaT, SiHa, Caski and HeLa cell lines. ${ }^{*} \mathrm{P}<0.05$ vs. HaCaT group, $\mathrm{n}=3$. (B) qPCR was used to detect NEAT1 expression in cervical cancer specimens and adjacent nontumor tissues. ${ }^{*} \mathrm{P}<0.05$ vs. normal group, $\mathrm{n}=20$. (C and D) Patients were divided into high NEAT1 group and low NEAT1 group according to the median value. Kaplan-Meier plots revealed that patients with high NEAT1 level had significantly worse overall survival $(\mathrm{P}=0.0245)$ and disease-free survival $(\mathrm{P}=0.0353)$.
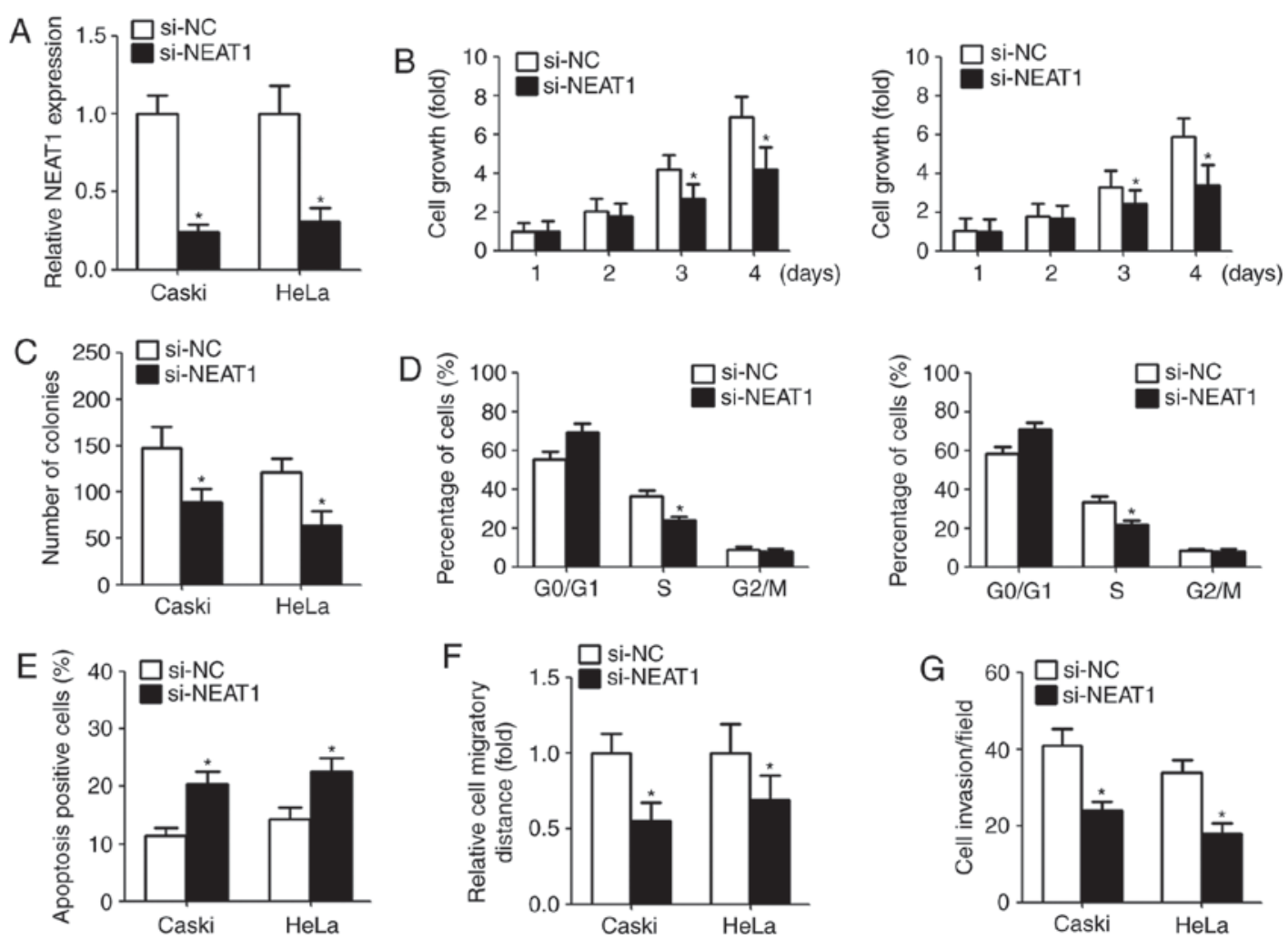

Figure 2. The effect of NEAT1 siRNA on cell proliferation, cell cycle, cell apoptosis, migration and invasion. Caski and HeLa cells were transfected with NEAT1-specific siRNA and control for $48 \mathrm{~h}$. (A) The relative expression of NEAT1 was detected by qPCR after transfection. (B) Cell viability was measured by using CCK-8 assay was at 1-4 day. (C) A colony-forming assay was performed after transfection. (D) Flow cytometry was used to determine the cell cycle profile. (E) Apoptosis rate was analyzed using flow cytometry stained with annexin V and propidium iodide. (F) Wound healing assay was used to determine migration after transfection. (G) Matrigel invasion assay was used to determine invasion after transfection. " $\mathrm{P}<0.05$ vs. control group, $\mathrm{n}=5$. 

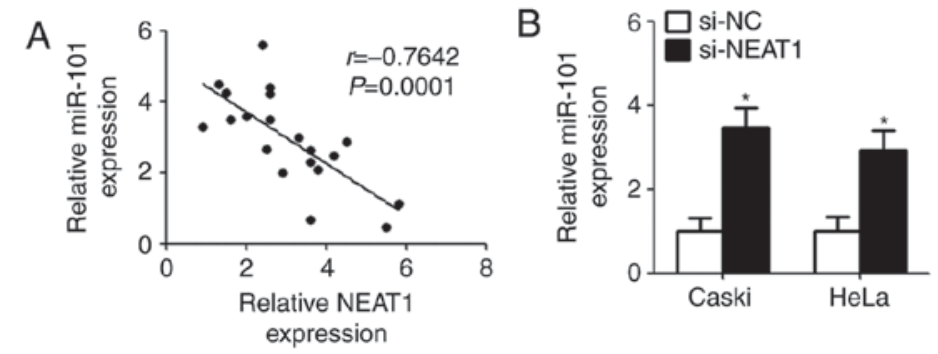

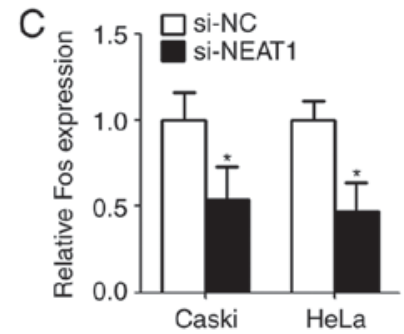

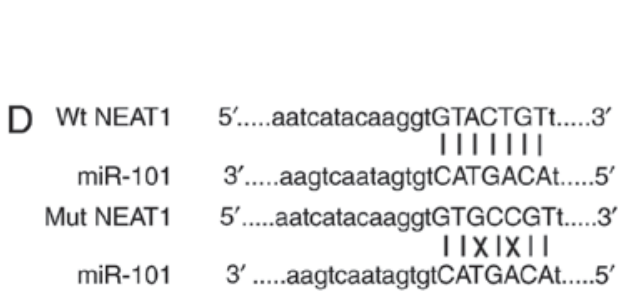

$\mathrm{E}$

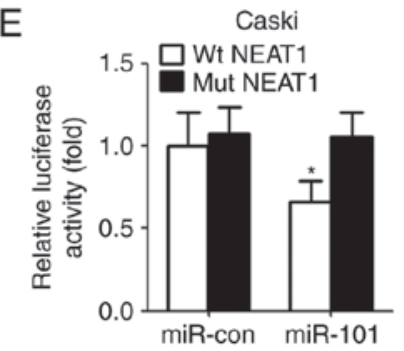

HeLa

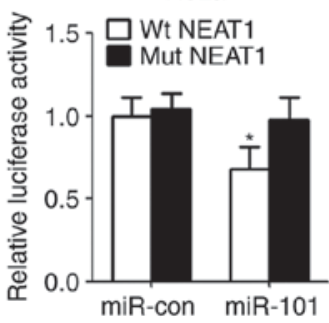

Figure 3. NEAT1 acts as a sponge of miR-101 by directly binding. (A) The correlation between NEAT1 level and miR-101 expression (n=20). Spearman's correlation coefficient $r=-0.7642, P=0.0001$. (B and C) $q P C R$ was used to detect the levels of miR-101 and Fos after transfection with NEAT1-specific siRNA and control for $48 \mathrm{~h}$. (D) Alignment of miR-101 sequence with wild type NEAT1 and NEAT1 mutated at the putative binding site by bioinformatics prediction. (E) Luciferase reporter activity in Caski and HeLa cells was detected after co-transfection miR-con/miR-101 mimics and luciferase vector (pmiR-GLo) containing the wild type/mutant NEAT1. ${ }^{*} \mathrm{P}<0.05$ vs. control group, $\mathrm{n}=5$.
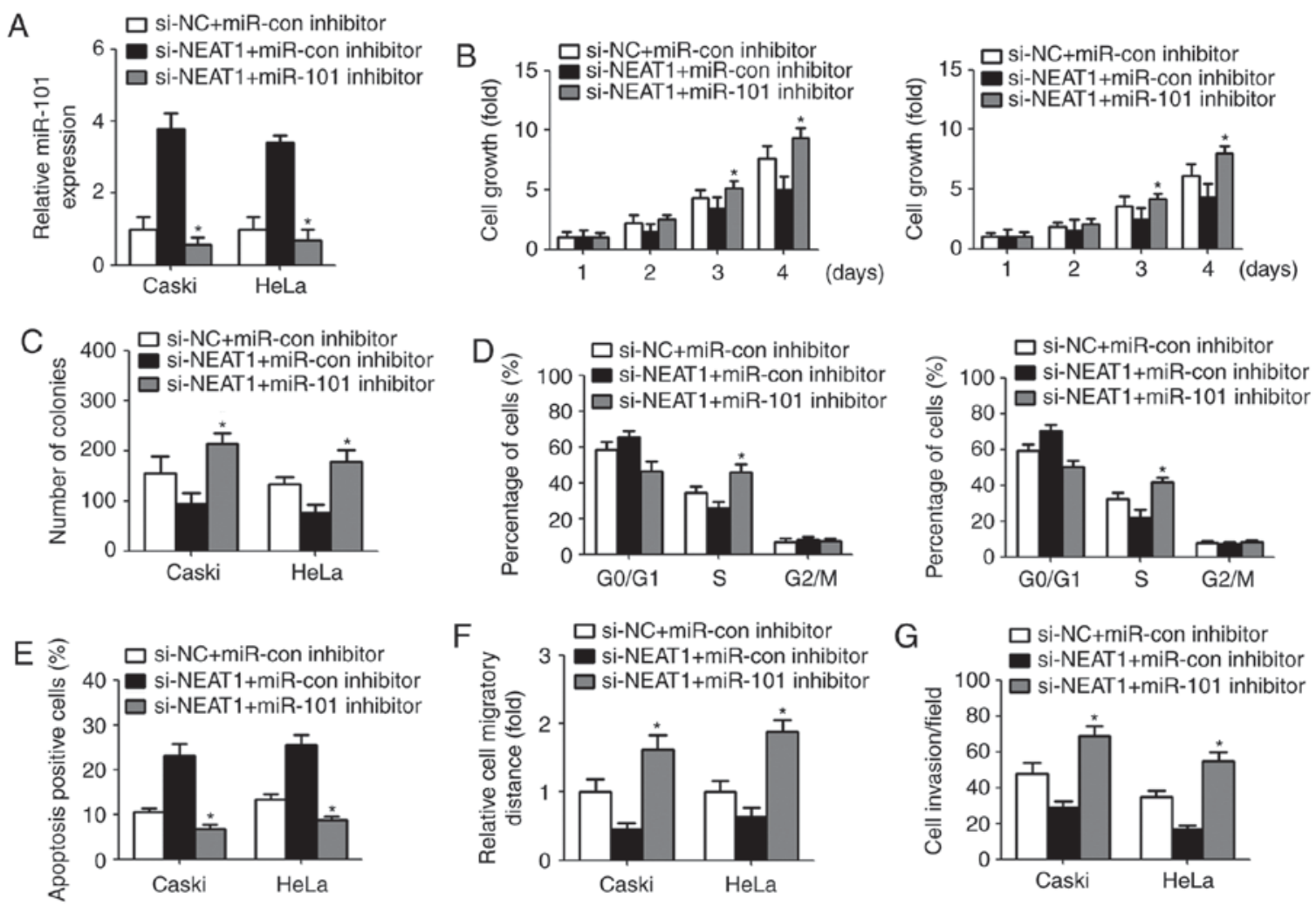

Figure 4. The biological effect of NEAT1 is through the negative modulation of miR-101. Caski and HeLa cells were co-transfected with NEAT1 siRNA and miR-101 inhibitor for 48 h. (A) Relative miR-101 level was measured by qPCR in cells. (B) CCK-8 assay was used to assess cell viability. (C) A colony-forming assay was performed after transfection. (D and E) Cell cycle and apoptosis rate were measured by flow cytometry. (F and G) Wound healing assay and matrigel invasion assay were used to determine migration and invasion, respectively. ${ }^{*} \mathrm{P}<0.05$ vs. si-NEAT1+miR-con inhibitor group, $\mathrm{n}=5$.

The inhibitory effect of NEAT1 on cervical cancer cells was reversed by miR-101 inhibitor. To investigate whether NEAT1 exert its biological effect by modulating miR-101, we repressed the miR-101 expression by transfection with miR-101 inhibitor in cervical cancer cells. qPCR analysis displayed a significant decreased miR-101 level after transfection with miR-101 inhibitor (Fig. 4A). CCK-8 assay revealed that downregulation of miR-101 restored NEAT1 siRNA-mediated inhibition of cell 
growth and colony-forming ability (Fig. 4B and C). Similarly, transfection with miR-101 inhibitor recovered the percentage of S phase and decreased cell apoptosis (Fig. 4D and E). Furthermore, NEAT1 siRNA-induced suppression of cell migration and invasion were abrogated due to miR-101 inhibitor transfection (Fig. 4F and G).

\section{Discussion}

Increasing evidences have implicated that aberrant expression of IncRNA is closely related to the occurrence and development of malignant tumors (10). Therefore, a further understanding of precise regulatory mechanism of lncRNA in cervical cancer progression is conductive to development of new diagnostic, more targeted, and effective, therapeutic treatments. NEAT1, an essential structural component of a nuclear domain called paraspeckles, is located on chromosome 11q13.1 and has been implicated in the development of corpus luteum and mammary gland as well as myeloid differentiation $(18,19)$. Cumulatively, recent studies indicate that NEAT1 was aberrantly expressed in a number of solid tumors, including non-small lung cancer, colorectal cancer, ovarian cancer, prostate cancer and hepatocellular cancer $(13,15,16,20,21)$. However, the molecular mechanisms of NEAT1 underlying the progression and development of malignancy remain poorly understood.

Our present study attempted to investigate the association between NEAT1 and the clinical characteristics in human cervical cancer. Previous studies reveal that NEAT1 is most abundant lncRNA in cervical tissue, and minor allele ' $\mathrm{C}$ ' of rs512715 in NEAT1 was associated with an increased risk of cervical cancer (22). In this study, our result demonstrated that NEAT1 level was upregulated in cervical cancer cells and tissues. Patients in the high NEAT1 group had significantly shorter overall/disease-free survival than those in the low NEAT1 group. It was confirmed to be an independent prognostic marker, suggesting that NEAT1 acts as a promoter in the development of cervical cancer.

In addition, our results demonstrated that NEAT1 knockdown inhibited cervical cancer cell growth via decreasing $S$ phase and increasing cell apoptosis. We also found that downregulation of NEAT1 inhibited the migratory and invasive ability of cervical cancer cells. These results are in accordance with above clinical data, indicating that NEAT1 is a novel booster in the progression of cervical cancer. NEAT1 have been widely investigated and plays an oncogenic role in various solid tumor. It is recognized that the mechanism by which NEAT1 mediates its function is complex and involves multiple factors, including sponging of tumor-suppressive microRNAs (23-25). miR-101 usually acts as a suppressor that inhibits the tumorigenicity of various cancer cell (26-29). Recent studies indicated that miR-101 is obviously downregulated in cervical cancer, miR-101 is a kind of miRNAs which act as a potential tumor suppressor in cervical cancer (30). Furthermore, miR-101 inhibits the G1-to-S phase transition of cervical cancer cells by targeting the proto-oncogene Fos (31).

Bioinformatics prediction indicated that NEAT1 may act as a miR-101 sponge. Our results demonstrated a significant negative correlation between NEAT1 expression and miR-101 level in clinical samples. In vitro, downregulation of NEAT1 elevated miR-101 level leading to a reduction of Fos expression. Luciferase reporter assay confirmed that NEAT1 could directly bind with miR-101 to control its expression in cervical cancer cells. Recent study suggests that NEAT1 promotes breast cancer cell growth and DNA synthesis through targeting miR-101. Our results lend credence to the previous study suggesting that NEAT1 interacted with miR-101 by directly targeting. Moreover, transfection with miR-101 inhibitor abolished NEAT1 siRNA-mediated effect on cell proliferation, apoptosis, migration and invasion. These results imply that NEAT1, acting as a miR-101 sponge, accelerate cervical cancer progression via suppressing miR-101.

In conclusion, current study found that NEAT1 acts a sponge of miR-101 in cervical cancer, and NEAT1 level is upregulated in cervical cancer, where it correlates with unfavorable clinicopathologic factors and poor prognosis. NEAT1 plays a crucial role in cervical cancer cell proliferation, apoptosis, migration and invasion targeting miR-101. Taken together, NEAT1 is an important molecular biomarker for predicting prognosis and a potential target for cervical cancer therapy.

\section{References}

1. Torre LA, Bray F, Siegel RL, Ferlay J, Lortet-Tieulent J and Jemal A: Global cancer statistics, 2012. CA Cancer J Clin 65: 87-108, 2015

2. Chen J, Fu Z, Ji C, Gu P, Xu P, Yu N, Kan Y, Wu X, Shen R and Shen Y: Systematic gene microarray analysis of the lncRNA expression profiles in human uterine cervix carcinoma. Biomed Pharmacother 72: 83-90, 2015.

3. Sakuragi N: Refining insight into cervical cancer progression. Lancet Oncol 15: 371-372, 2014.

4. Schiffman M, Wentzensen N, Wacholder S, Kinney W, Gage JC and Castle PE: Human papillomavirus testing in the prevention of cervical cancer. J Natl Cancer Inst 103: 368-383, 2011.

5. Baak JP, Kruse AJ, Robboy SJ, Janssen EA, van Diermen B and Skaland I: Dynamic behavioural interpretation of cervical intraepithelial neoplasia with molecular biomarkers. J Clin Pathol 59: 1017-1028, 2006.

6. Lee M, Kim HJ, Kim SW, Park SA, Chun KH, Cho NH, Song YS and Kim YT: The long non-coding RNA HOTAIR increases tumour growth and invasion in cervical cancer by targeting the Notch pathway. Oncotarget 7: 44558-44571, 2016.

7. Mattick JS: The genetic signatures of noncoding RNAs. PLoS Genet 5: e1000459, 2009.

8. Gibb EA, Brown CJ and Lam WL: The functional role of long non-coding RNA in human carcinomas. Mol Cancer 10: 38, 2011.

9. Mercer TR, Dinger ME and Mattick JS: Long non-coding RNAs: Insights into functions. Nat Rev Genet 10: 155-159, 2009.

10. Calore F, Lovat F and Garofalo M: Non-coding RNAs and cancer. Int J Mol Sci 14: 17085-17110, 2013.

11. Zhang J, Liu SC, Luo XH, Tao GX, Guan M, Yuan H and Hu DK: Exosomal long noncoding RNAs are differentially expressed in the cervicovaginal lavage samples of cervical cancer patients. J Clin Lab Anal 30: 1116-1121, 2016.

12. Gibb EA, Becker-Santos DD, Enfield KS, Guillaud M, Niekerk Dv, Matisic JP, Macaulay CE and Lam WL: Aberrant expression of long noncoding RNAs in cervical intraepithelial neoplasia. Int J Gynecol Cancer 22: 1557-1563, 2012.

13. Jen J, Tang YA, Lu YH, Lin CC, Lai WW and Wang YC: Oct4 transcriptionally regulates the expression of long non-coding RNAs NEAT1 and MALAT1 to promote lung cancer progression. Mol Cancer 16: 104, 2017.

14. Chen X, Kong J, Ma Z, Gao S and Feng X: Up regulation of the long non-coding RNA NEAT1 promotes esophageal squamous cell carcinoma cell progression and correlates with poor prognosis. Am J Cancer Res 5: 2808-2815, 2015.

15. Peng W, Wang Z and Fan H: LncRNA NEAT1 impacts cell proliferation and apoptosis of colorectal cancer via regulation of akt signaling. Pathol Oncol Res 23: 651-656, 2017. 
16. Mang Y, Li L, Ran J, Zhang S, Liu J, Li L, Chen Y, Liu J, Gao Y and Ren G: Long noncoding RNA NEAT1 promotes cell proliferation and invasion by regulating hnRNP A2 expression in hepatocellular carcinoma cells. Onco Targets Ther 10: 1003-1016, 2017.

17. Ergun S and Oztuzcu S: Oncocers: ceRNA-mediated cross-talk by sponging miRNAs in oncogenic pathways. Tumour Biol 36: 3129-3136, 2015.

18. Clemson CM, Hutchinson JN, Sara SA, Ensminger AW, Fox AH, Chess A and Lawrence JB: An architectural role for a nuclear noncoding RNA: NEAT1 RNA is essential for the structure of paraspeckles. Mol Cell 33: 717-726, 2009.

19. Sasaki YT, Ideue T, Sano M, Mituyama T and Hirose T, MENepsilon/beta noncoding RNAs are essential for structural integrity of nuclear paraspeckles. Proc Natl Acad Sci USA 106: 2525-2530, 2009.

20. Chen ZJ, Zhang Z, Xie BB and Zhang HY: Clinical significance of up-regulated lncRNA NEAT1 in prognosis of ovarian cancer. Eur Rev Med Pharmacol Sci 20: 3373-3377, 2016.

21. Chakravarty D, Sboner A, Nair SS, Giannopoulou E, Li R, Hennig S, Mosquera JM, Pauwels J, Park K, Kossai M, et al: The oestrogen receptor alpha-regulated lncRNA NEAT1 is a critical modulator of prostate cancer. Nat Commun 5: 5383, 2014.

22. Fang J, Li Y, Zhang J, Yan M, Li J, Bao S and Jin T: Correlation between polymorphisms in microRNA-regulated genes and cervical cancer susceptibility in a Xinjiang Uygur population. Oncotarget 8: 31758-31764, 2017.

23. Qian K, Liu G, Tang Z, Hu Y, Fang Y, Chen Z and Xu X: The long non-coding RNA NEAT1 interacted with miR-101 modulates breast cancer growth by targeting EZH2. Arch Biochem Biophys 615: 1-9, 2017.
24. Li JH, Zhang SQ, Qiu XG, Zhang SJ, Zheng SH and Zhang DH: Long non-coding RNA NEAT1 promotes malignant progression of thyroid carcinoma by regulating miRNA-214. Int J Oncol 50: 708-716, 2017.

25. Huang B, Liu C, Wu Q, Zhang J, Min Q, Sheng T, Wang X and Zou Y: Long non-coding RNA NEAT1 facilitates pancreatic cancer progression through negative modulation of miR-506-3p. Biochem Biophys Res Commun 482: 828-834, 2017.

26. Jiang R, Zhang C, Liu G, Gu R and Wu H: MicroRNA-101 inhibits proliferation, migration and invasion in osteosarcoma cells by targeting ROCK1. Am J Cancer Res 7: 88-97, 2017.

27. Wang L, Yao J, Sun H, He K, Tong D, Song T and Huang C: MicroRNA-101 suppresses progression of lung cancer through the PTEN/AKT signaling pathway by targeting DNA methyltransferase 3A. Oncol Lett 13: 329-338, 2017.

28. Liu J, Pang Y, Wang H, Li Y, Sun X, Xu F, Ren H and Liu D: miR-101 inhibits the proliferation and migration of breast cancer cells via downregulating the expression of DNA methyltransferase 3a. Xi Bao Yu Fen Zi Mian Yi Xue Za Zhi 32: 299-303, 2016 (In Chinese)

29. Zheng HB, Zheng XG and Liu BP: miRNA-101 inhibits ovarian cancer cells proliferation and invasion by down-regulating expression of SOCS-2. Int J Clin Exp Med 8: 20263-20270, 2015.

30. Huang F, Lin C, Shi YH and Kuerban G: MicroRNA-101 inhibits cell proliferation, invasion and promotes apoptosis by regulating cyclooxygenase-2 in Hela cervical carcinoma cells. Asian Pac J Cancer Prev 14: 5915-5920, 2013.

31. Liang X, Liu Y, Zeng L, Yu C, Hu Z, Zhou Q and Yang Z: miR-101 inhibits the G1-to-S phase transition of cervical cancer cells by targeting Fos. Int J Gynecol Cancer 24: 1165-1172, 2014. 